\title{
Precise BER Formulas for Asynchronous QPSK-Modulated DS-CDMA Systems Using Random Quaternary Spreading Over Rayleigh Channels
}

\author{
Xiang Liu and Lajos Hanzo
}

\begin{abstract}
Precise bit-error-ratio (BER) analysis of an asynchronous QPSK-modulated direct-sequence code-division multiple-access system using random quaternary spreading sequences for transmission over Rayleigh channels is performed based on the characteristic-function approach. Its accuracy is verified by our numerical simulation results and also compared with those of the Gaussian approximation.
\end{abstract}

Index Terms-Asynchronous direct-sequence code-division multiple-access (DS-CDMA), bit-error-ratio (BER), precise, QPSK, quarternary spreading, Rayleigh.

\section{INTRODUCTION}

$\mathbf{T}$ RADITIONALLY, the in-phase and quadrature-phase components of QPSK-modulated direct-sequence codedivision multiple-access (DS-CDMA) systems are separately spread [1]. Quaternary spreading sequences have been applied in the wideband CDMA systems characterized in [2]. As a benefit of their relative simplicity compared with other polyphase sequences [3], a number of quaternary spreading sequences, which are synonymously also referred to as complex-valued spreading sequences, have been proposed in [4]-[6]. Some of them were claimed to outperform gold and $m$-sequences in asynchronous DS-CDMA systems [5], [6]. A receiver architecture using quaternary spreading sequences and QPSK modulation was proposed in [7].

The bit-error-ratio (BER) performance of the family of DS-CDMA systems using quaternary spreading and QPSK modulation has been investigated using a variety of techniques, including precise analysis [6], [7], approximations [6], [8], [9], bounding techniques [9], or simulations [5], [8]. The achievable BER performance over both additive white Gaussian noise (AWGN) [5], [7]-[9] and Rayleigh [6] channels was also documented. The BER performance attainable using

Manuscript received October 4, 2005; revised October 29, 2006 and May 8, 2007. This work was supported in part by the Engineering and Physical Sciences Research Council (EPSRC), U.K., and in part by the EU under the auspices of the NEWCOM and Phoenix Projects. This paper was presented at the IEEE Wireless Communications and Networking Conference 2006, Las Vegas, NV. The review of this paper was coordinated by Prof. H. Leib.

X. Liu is with the University of Southampton, SO17 1BJ Southampton, U.K., and also with ArrayComm LLC, GU2 7YD Guildford, U.K.

L. Hanzo is with the University of Southampton, SO17 1BJ Southampton, U.K.

Digital Object Identifier 10.1109/TVT.2007.905257 both deterministic quaternary spreading sequences [5]-[7], [9] and random quaternary spreading sequences [8] was also studied.

In contrast to the performance analysis of [6] and [7], we provide the precise BER analysis of the asynchronous DS-CDMA systems using the random quaternary spreading sequences and the QPSK modulation for transmission over the Rayleigh channels rather than employing the deterministic quaternary spreading sequences [6], [7] for transmission over the AWGN channels [7]. Furthermore, we do not assume the independence between the real and imaginary parts of the received signal, which was implicitly assumed in [7] when calculating symbol error ratio. In contrast to the performance analysis of [9], we use quadriphase data rather than binary data.

\section{System Model}

We consider a general asynchronous QPSK-modulated DSCDMA system using the random quaternary spreading and a rectangular chip waveform for transmission over a Rayleighfading channel. We assume that there are $K$ simultaneously transmitting users, and the zeroth user's signal is the desired one. Since exactly the same system model has been described in [7], we commence our discussions from the decision statistics $\widetilde{Z}$ of the received signal at the output of the correlation receiver matched to the zeroth user's signal

$$
\widetilde{Z}=h_{0} L \widetilde{b}_{0,0}+\sum_{k=1}^{K-1} \widetilde{X}_{k} h_{k} e^{j \Delta_{k}}+\widetilde{\eta}
$$

where $L$ is the length of the spreading sequences. The signal amplitudes $\left\{h_{k}\right\}_{k=0}^{K-1}$ of the $k$ th user are assumed to be independently Rayleigh-distributed with a second uncentered moment of $\left\{2 \sigma_{k}^{2}\right\}$, whereas the quaternary data sequences $\left\{\widetilde{b}_{k, m}\right\}_{m=-\infty}^{\infty}$ are assumed to be mutually independent and are uniformly distributed with a probability of $P\left\{\widetilde{b}_{k, m}=\right.$ $\pm 1 \pm j\}=1 / 4$. Finally, $\widetilde{\eta}$ is the noise component, which is a zero-mean complex-valued Gaussian variable. The phaseshift difference $\left\{\Delta_{k}\right\}_{k=1}^{K-1}$ between the $k$ th and zeroth users is defined as $\Delta_{k}=-\omega_{\mathrm{c}}\left(\tau_{k}-\tau_{0}\right)+\left(\theta_{k}-\theta_{0}\right)$, where $\omega_{\mathrm{c}}$ is the common carrier frequency, $T_{\mathrm{c}}$ is the chip duration, and the phase shifts $\left\{\theta_{k}\right\}_{k=0}^{K-1}$ and the time delays $\left\{\tau_{k}\right\}_{k=0}^{K-1}$ are 
independently and uniformly distributed in $[0,2 \pi)$ and $\left[0, L T_{\mathrm{c}}\right)$, respectively. The random variable $\widetilde{X}_{k}$ may be further expressed as [10], [11]

$$
\begin{aligned}
\widetilde{X}_{k}=\sum_{m=0}^{L-2} \widetilde{Y}_{k, m}\left[\left(1-\nu_{k}\right)\right. & \left.+\frac{1}{2} \widetilde{a}_{0, m} \widetilde{a}_{0, m+1}^{*} \nu_{k}\right] \\
& +\widetilde{Y}_{k, L-1} \nu_{k}+\widetilde{Y}_{k, L}\left(1-\nu_{k}\right)
\end{aligned}
$$

where the $(L+1)$ random variables $\left\{\widetilde{Y}_{k, m}\right\}_{m=0}^{L}$ are mutually independent and uniformly distributed, which are conditioned on the zeroth user's spreading sequence $\left\{\widetilde{a}_{0, m}\right\}_{m=0}^{L-1}$. Furthermore, the relative chip shifts $\left\{\nu_{k}\right\}_{k=1}^{K-1}$ between the $k$ th and zeroth users, which are normalized by the chip duration, are mutually independent and uniformly distributed in $[0,1)[10],[11]$.

\section{BER ANALYSIS}

The $(L-1)$ possible chip combinations of $\left\{(1 / 2) \widetilde{a}_{0, m}\right.$ $\left.\widetilde{a}_{0, m+1}^{*}\right\}_{m=0}^{L-2}$ can be categorized into four sets according to the relative change of the chip value. Let $A, B, C$, and $D$ denote the number of the relative phase changes of $0^{\circ}, 180^{\circ}$, $270^{\circ}$, and $90^{\circ}$ between the adjacent two chips' values within the zeroth user's spreading sequence, respectively. Then, we have $A+B+C+D=L-1$. Upon defining the cochannel interference (CCI) $\widetilde{I}_{k}=\widetilde{X}_{k} h_{k} e^{j \Delta_{k}}$ incurred by the $k$ th user, the characteristic function of $\Re\left\{\widetilde{I}_{k}\right\}$ conditioned on $A, B$, and $C$ can be expressed as

$$
\begin{aligned}
\Phi_{\Re\left\{\widetilde{I}_{k}\right\} \mid A, B, C}(\omega) & 2^{-2(L+1)} \sum_{\widetilde{d}_{1} \in \mathcal{A}} \sum_{\widetilde{d}_{2} \in \mathcal{B}} \sum_{\widetilde{d}_{3} \in \mathcal{C}} \sum_{\widetilde{d}_{4} \in \mathcal{D}} \\
& \times\left(\begin{array}{c}
A \\
\frac{\Re\left\{\widetilde{d}_{1}\right\}+A}{2}
\end{array}\right)\left(\begin{array}{c}
A \\
\frac{\Im\left\{\widetilde{d}_{1}\right\}+A}{2}
\end{array}\right)\left(\begin{array}{c}
B \\
\frac{\Re\left\{\widetilde{d}_{2}\right\}+B}{2}
\end{array}\right)\left(\begin{array}{c}
B \\
\frac{\left.\Im \widetilde{d}_{2}\right\}+B}{2}
\end{array}\right) \\
& \times\left(\begin{array}{c}
C \\
\frac{\Re\left\{\widetilde{d}_{3}\right\}+C}{2}
\end{array}\right)\left(\begin{array}{c}
C \\
\frac{\Im\left\{\widetilde{d}_{3}\right\}+C}{2}
\end{array}\right)\left(\begin{array}{c}
D \\
\frac{\Re\left\{\widetilde{d}_{4}\right\}+D}{2}
\end{array}\right)\left(\begin{array}{c}
D \\
\frac{\Im\left\{\widetilde{d}_{4}\right\}+D}{2}
\end{array}\right) \\
& \times \sum_{\widetilde{Y}_{k, L-1}, \widetilde{Y}_{k, L}} \Phi_{\Re\left\{\widetilde{I}_{k}\right\} \mid \lambda_{0}, \lambda_{1}, \lambda_{2}}\left(\sigma_{k} \omega\right)
\end{aligned}
$$

where the sets $\mathcal{A}, \mathcal{B}, \mathcal{C}$, and $\mathcal{D}$ are defined as

$$
\begin{aligned}
& \mathcal{A}=\left\{\widetilde{d}_{1} \mid \Re\left\{\widetilde{d}_{1}\right\}, \Im\left\{\widetilde{d}_{1}\right\} \in\{-A,-(A-2), \ldots, A\}\right\} \\
& \mathcal{B}=\left\{\widetilde{d}_{2} \mid \Re\left\{\widetilde{d}_{2}\right\}, \Im\left\{\widetilde{d}_{2}\right\} \in\{-B,-(B-2), \ldots, B\}\right\} \\
& \mathcal{C}=\left\{\widetilde{d}_{3} \mid \Re\left\{\widetilde{d}_{3}\right\}, \Im\left\{\widetilde{d}_{3}\right\} \in\{-C,-(C-2), \ldots, C\}\right\} \\
& \mathcal{D}=\left\{\widetilde{d}_{4} \mid \Re\left\{\widetilde{d}_{4}\right\}, \Im\left\{\widetilde{d}_{4}\right\} \in\{-D,-(D-2), \ldots, D\}\right\} .
\end{aligned}
$$

The function $\Phi_{\Re\left\{\widetilde{I}_{k}\right\} \mid \lambda_{0}, \lambda_{1}, \lambda_{2}}(\omega)$ is defined as (5), shown at the bottom of the page, where the coefficients $\lambda_{0}, \lambda_{1}$, and $\lambda_{2}$ are given by

$$
\begin{aligned}
\lambda_{0}=\mid \widetilde{d}_{1} & +\widetilde{d}_{2}+\widetilde{d}_{3}+\widetilde{d}_{4}+\left.\widetilde{Y}_{k, L}\right|^{2} \\
\lambda_{1}=\Re\{ & \left(\widetilde{d}_{1}+\widetilde{d}_{2}+\widetilde{d}_{3}+\widetilde{d}_{4}+\widetilde{Y}_{k, L}\right) \\
& \left.\quad \times\left[-2 \widetilde{d}_{2}-(1-j) \widetilde{d}_{3}-(1+j) \widetilde{d}_{4}+\widetilde{Y}_{k, L-1}-\widetilde{Y}_{k, L}\right]^{*}\right\}
\end{aligned}
$$$$
\lambda_{2}=\left|-2 \widetilde{d}_{2}-(1-j) \widetilde{d}_{3}-(1+j) \widetilde{d}_{4}+\widetilde{Y}_{k, L-1}-\widetilde{Y}_{k, L}\right|^{2} .
$$

Since the CCI $\left\{\widetilde{I}_{k}\right\}_{k=1}^{K-1}$ conditioned on $A, B$, and $C$ is independent [10], [11], the BER of the zeroth user's real component conditioned on $A, B$, and $C$ can be shown as [10]

$$
\begin{aligned}
P_{e \mid A, B, C}^{\mathrm{r}}=\frac{1}{2}-\frac{\sigma_{0} L}{\sqrt{2 \pi}} \int_{0}^{\infty} \exp & {\left[-\frac{1}{2} \omega^{2}\left(\sigma_{0}^{2} L^{2}+\sigma_{\Re\{\widetilde{\eta}\}}^{2}\right)\right] } \\
\times & \times \prod_{k=1}^{K-1} \Phi_{\Re\left\{\widetilde{I}_{k}\right\} \mid A, B, C}(\omega) d \omega .
\end{aligned}
$$

Then, the BER of the zeroth user's real component is obtained by averaging $P_{e \mid A, B, C}^{\mathrm{r}}$ over all spreading sequences [10]. Following the same approach, we may conclude that the average BER of the zeroth user's imaginary component $P_{e}^{\mathrm{i}}$ has the same value as $P_{e}^{\mathrm{r}}$. Finally, we arrive at the overall BER $P_{e}$ averaged over both the real and imaginary components of the zeroth user yielding

$$
\begin{aligned}
P_{e}=P_{e}^{\mathrm{r}}= & 4^{-(L-1)} \sum_{A=0}^{L-1} \sum_{B=0}^{L-1-A} \sum_{C=0}^{L-1-A-B}\left(\begin{array}{c}
L-1 \\
A
\end{array}\right) \\
& \times\left(\begin{array}{c}
L-1-A \\
B
\end{array}\right)\left(\begin{array}{c}
L-1-A-B \\
C
\end{array}\right) P_{e \mid A, B, C}^{\mathrm{r}} .
\end{aligned}
$$

$$
\Phi_{\Re\left\{\widetilde{I}_{k}\right\} \mid \lambda_{0}, \lambda_{1}, \lambda_{2}}(\omega)= \begin{cases}\exp \left(-\frac{1}{2} \lambda_{0} \omega^{2}\right), & \text { if } \lambda_{2}=0 \\ \frac{1}{\omega} \sqrt{\frac{\pi}{2 \lambda_{2}}} \exp \left[\frac{1}{2}\left(\frac{\lambda_{1}^{2}}{\lambda_{2}}-\lambda_{0}\right) \omega^{2}\right] & \\ \quad \times\left\{\operatorname{erfc}\left(\frac{\lambda_{1} \omega}{\sqrt{2 \lambda_{2}}}\right)-\operatorname{erfc}\left[\sqrt{\frac{\lambda_{2}}{2}}\left(1+\frac{\lambda_{1}}{\lambda_{2}}\right) \omega\right]\right\}, & \text { if } \lambda_{2} \neq 0\end{cases}
$$




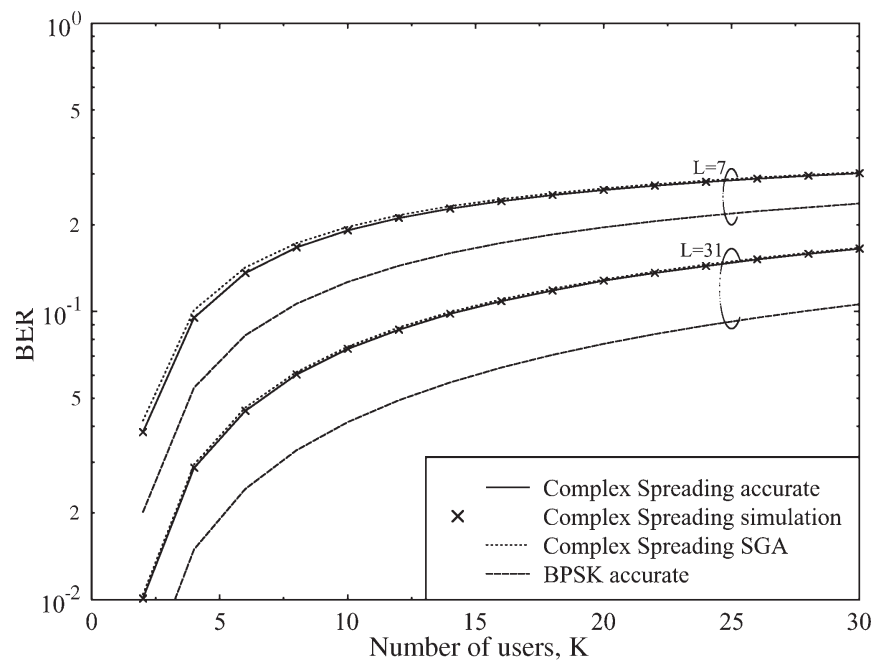

Fig. 1. BER versus the number of users $K$ in the asynchronous QPSKmodulated DS-CDMA system using the random quaternary spreading sequences. The lengths of the random quaternary spreading sequences are $L=7$ and 31 , respectively, the average power of all users at the receiver is equal, and the background noise is ignored, i.e., when $\gamma_{\mathrm{SNR}}=\infty$.

\section{NUMERICAL RESULtS}

We will compare the BER results acquired by our precise analysis derived in Section III with that of the standard Gaussian approximation (SGA) [10], [11], with that of the BPSK system of [10], and with our simulations described in this section. Similar to the discussions in [10] and [11], the complexity of our analysis, the SGA, and the simulation is on the order of $\Theta\left(L^{3}\right), \Theta(1)$, and $\Theta\left(4^{L}\right)$, respectively, where $\Theta$ is the big-Theta notation. ${ }^{1}$ Fig. 1 shows that the results obtained by our precise analysis exactly match those obtained by simulations for two different-length random spreading sequences when using $L=7$ and 31. However, the SGA overestimates the BER, particularly in the scenario where there is a low number of interfering users and when short spreading sequences are used. The BER of the QPSK system is higher than that of the BPSK systems due to the crosstalk between the real and imaginary components. Fig. 2 also confirms that the BER results obtained by our precise analysis match those obtained by simulation for both of the different-length random spreading sequences, i.e., for $L=7$ and 31. By contrast, the SGA slightly overestimates the BER, particularly when the SNR is high and short spreading sequences are used. The BER of the QPSK systems is higher than that of the BPSK systems due to the crosstalk between the real and imaginary components.

\section{CONCLUSION}

In this paper, we provided exact BER formulas for the asynchronous QPSK-modulated DS-CDMA system using the random quaternary spreading sequences for transmission over

\footnotetext{
${ }^{1} \mathrm{~A}$ function is in $\Theta(f(n))$ if it does not significantly increase faster or slower than $f(n)$, i.e., there exist two positive real constants $c_{1}$ and $c_{2}$ and a positive integer $n_{0}$ so that $c_{1} n \leq \Theta(f(n)) \leq c_{2} n$ for $\forall n \geq n_{0}$.
}

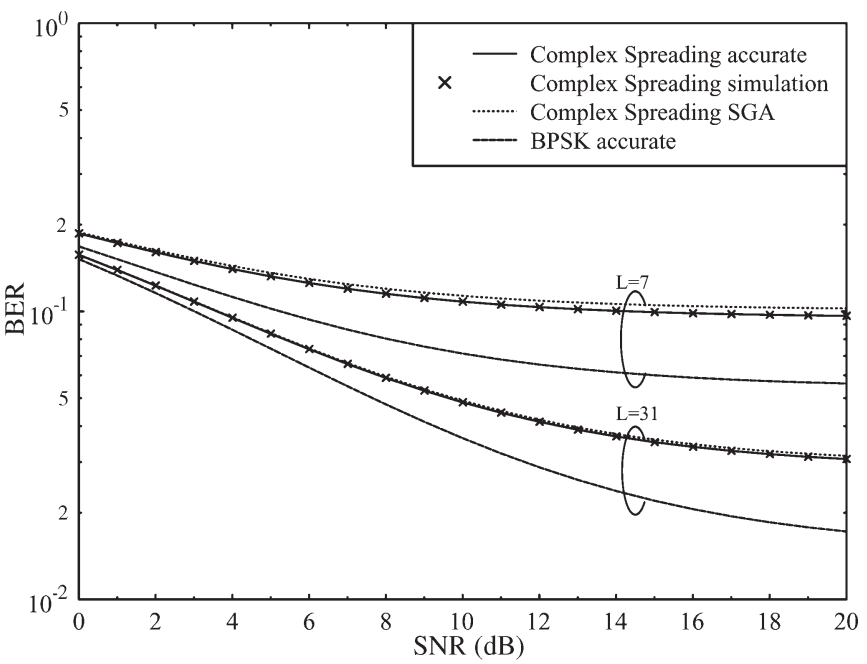

Fig. 2. BER versus per-bit SNR in the asynchronous QPSK-modulated DS-CDMA system using the random quaternary spreading sequences. The lengths of the random quaternary spreading sequences are $L=7$ and 31 , respectively, and the average power of all users at the receiver is equal. The number of users is $K=4$.

the Rayleigh channels. Its accuracy was verified by our numerical simulation results and also compared with those of the Gaussian approximation.

\section{ACKNOWLEDGMENT}

The authors would like to thank F.-C. Kuo for her valuable suggestions that help improve this paper. The authors would also like to thank the anonymous reviewers for the helpful critique.

\section{REFERENCES}

[1] F. D. Garber and M. B. Pursley, "Performance of offset quadriphase spread-spectrum multiple-access communications," IEEE Trans. Commun., vol. COM-29, no. 3, pp. 305-314, Mar. 1981.

[2] 3GPP TS 25.213 V6.3.0. (2005, Jun.). Spreading and Modulation (FDD). 3rd Generation Partnership Project. [Online]. Available: http://www. 3gpp.org/

[3] P. Fan and M. Darnell, Sequence Design for Communications Applications. Hoboken, NJ: Wiley, 1996.

[4] S. R. Park, I. Song, S. Yoon, and J. Lee, "A new polyphase sequence with perfect even and good odd cross-correlation functions for DS/CDMA systems," IEEE Trans. Veh. Technol., vol. 51, no. 5, pp. 855-866, Sep. 2002.

[5] S. Rahardja, W. Ser, and Z. Lin, "UCHT-based complex sequences for asynchronous CDMA system," IEEE Trans. Commun., vol. 51, no. 4, pp. 618-626, Apr. 2003.

[6] S. Xie and S. Rahardja, "Performance evaluation for quaternary DS-SSMA communications with complex signature sequences over Rayleigh-fading channels," IEEE Trans. Wireless Commun., vol. 4, no. 1, pp. 266-277, Jan. 2005.

[7] T. G. Macdonald and M. B. Pursley, "The performance of direct-sequence spread spectrum with complex processing and quaternary data modulation," IEEE J. Sel. Areas Commun., vol. 18, no. 8, pp. 1408-1417, Aug. 2000.

[8] J. H. Cho, Y. K. Jeong, and J. S. Lehnert, "Average bit-error-rate performance of band-limited DS/SSMA communications," IEEE Trans. Commun., vol. 50, no. 7, pp. 1150-1159, Jul. 2002.

[9] A. Lam and F. Ozluturk, "Performance bounds for DS/SSMA communications with complex signature sequences," IEEE Trans. Commun., vol. 40, no. 10, pp. 1607-1614, Oct. 1992. 
[10] J. Cheng and N. Beaulieu, "Accurate DS-CDMA bit-error probability calculation in Rayleigh fading," IEEE Trans. Wireless Commun., vol. 1, no. 1, pp. 3-15, Jan. 2002.

[11] J. S. Lehnert and M. B. Pursley, "Error probabilities for binary directsequence spread-spectrum communications with random signature sequences," IEEE Trans. Commun., vol. COM-35, no. 1, pp. 87-98, Jan. 1987.

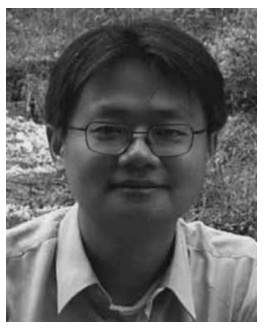

Xiang Liu is currently working toward the Ph.D. degree in electronics at the University of Southampton, Southampton, U.K.

$\mathrm{He}$ is currently a Software Engineer with ArrayComm LLC, Guildford, U.K.

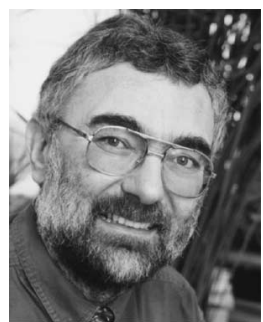

Lajos Hanzo received the degree in electronics in 1976 and the Ph.D. degree in 1983.

During his 31-year career in telecommunications, he has held various research and academic posts in Hungary, Germany, and the U.K. Since 1986, he has been with the School of Electronics and Computer Science, University of Southampton, Southampton, U.K., where he is the Chair in telecommunications. Currently, he is directing an academic research team, working on a range of research projects in the field of wireless multimedia communications sponsored by industry, the Engineering and Physical Sciences Research Council, U.K., the European IST Programme, and the Mobile Virtual Centre of Excellence, U.K. $\mathrm{He}$ is a coauthor of 15 books on mobile radio communications, totaling more than 10000 pages, and has published more than 700 research papers. He is an enthusiastic supporter of industry and academia, and he offers a range of industrial courses.

Dr. Hanzo is an IEEE Distinguished Lecturer of both the Communications Society (ComSoc) and the IEEE Vehicular Technology Society (VTS). He is also the Governor of both ComSoc and the VTS. He was the Technical Program Committee Chair of several IEEE conferences, has presented keynote lectures, and is the recipient of a number of distinctions. He received the ComSoc WTV Achievement Award in 2007. 